
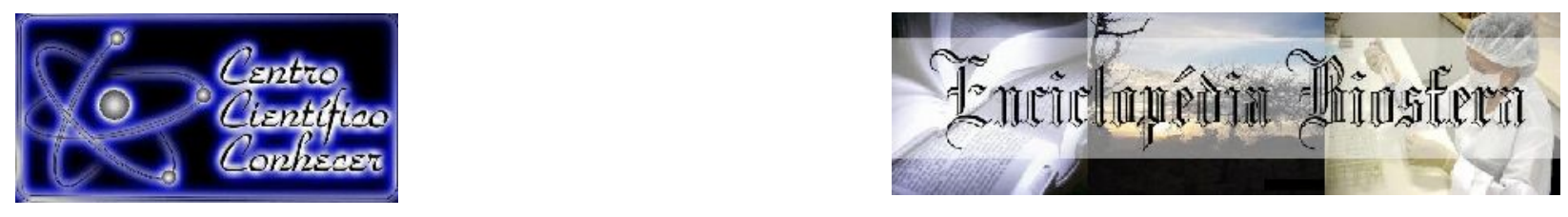

\title{
PERFIL DE TRINTA CÃES VÍTIMAS DE MAUS TRATOS RESGATADOS POR PROTETORES DE ANIMAIS
}

\author{
Maristela dos Santos Vieira ${ }^{1}$; Dnilson Carlos Dias ${ }^{1}$, Viviane Aguiar de Souza ${ }^{1}$; \\ Regiani Nascimento Gagno Pôrto ${ }^{2}$, Ana Paula Iglesias Santin ${ }^{2}$ \\ ${ }^{1}$ Acadêmico(a) em Medicina Veterinária da Universidade Federal de Goiás, Campus \\ Samambaia, Goiânia, Brasil (maristela.santosv@gmail.com) \\ ${ }^{2}$ Professor Associado da Escola de Veterinária e Zootecnia da Universidade Federal \\ de Goiás, Campus Samambaia, Goiânia, Brasil
}

\section{Recebido em: 06/04/2019 - Aprovado em: 10/06/2019 - Publicado em: 30/06/2019 DOI: 10.18677/EnciBio_2019A99}

\begin{abstract}
RESUMO
Os maus tratos contra cães é uma notícia frequentemente abordada em diversas mídias sociais, entretanto, são raros os estudos que visam a compreensão dos aspectos ligados a esta prática, da identificação do tipo de maus tratos e das características dos cães acometidos. Considerando estes aspectos, o presente estudo visou a identificação da raça, sexo, porte e idade dos cães que sofreram maus tratos, os principais tipos de maus tratos praticados e a existência de associação entre os dados obtidos. Para tanto, realizou-se exames anatomopatológicos e a análise de 30 casos em que se houve a confirmação de maus tratos, considerando os tópicos previstos na legislação. Os dados foram submetidos ao teste Qui-quadrado de aderência e o Teste-G. Identificou-se que os cães vítimas de maus tratos normalmente são SRD, adultos e de médio ou pequeno porte. Além disso, pode-se constatar que o abandono é o tipo de maus tratos mais frequente, sendo que os animais normalmente são resgatados da rua. Em relação aos animais com lesões, a maioria destes apresentavam dermatopatias. Não foram identificadas diferenças significativas entre o sexo, estado nutricional e presença de ectoparasitas entre os animais resgatados.
\end{abstract}

PALAVRAS- CHAVE: Abandono, Abuso, Canino, Violência.

\section{MISTREATMENT DOGS PROFILE}

Dog mistreatment is a frequent news in several social media, however it is rare to find studies understanding the aspects related to this practice, identification of the abuse type and the characteristics of the dogs victims of these practices. Considering these aspects, the present study aims to identify the breed, sex, size and age of the dogs who suffered abuse, the main types of maltreatment practiced and the existence of an association between the obtained data. For this purpose, anatomopathological examinations were performed in 30 cases of mistreatment. The datas were analyzed by Chi-Square test and G-test. In this study, the dogs were usually mongrel, adult and medium or small size. Abandonment is the most frequent abuse against dogs, and animals were usually rescued from the street. The most common lesions observed in these animals were dermatopathies. No significant 
differences were identified between sex, nutritional status and presence of ectoparasites in rescued dogs.

KEYWORDS: Abandonment, Abuse, Canine, Violence.

\section{INTRODUÇÃO}

Estudos indicam que a domesticação do cão ocorreu em parte na Europa pela população de caçadores e coletores. Inicialmente a relação entre o homem e o cão era basicamente utilitária, no entanto, no decorrer do tempo houve o favorecimento da reprodução de cães bem adaptados às condições locais e que apresentavam traços preferenciais ao homem (OLLIVER, 2017). As mudanças nos hábitos sociais e culturais da população levaram ao surgimento de laços afetivos entre os animais de estimação e seus tutores (RODRIGUES et al., 2017). Este foi um fator desencadeante da modificação das relações entre o homem e os "pets". A partir desta mudança na relação, a convivência e a estimação tornaram-se os principais motivos da criação destes animais. Atualmente, os animais de estimação como os cães adquirem frequentemente posições elevadas dentro das estruturas sociais das famílias (ABONISIO; BAPTISTELLA, 2016), sendo declarados integrantes do que se considera um novo modelo familiar (RODRIGUES et al., 2017).

Alguns estudos indicam vários benefícios na interação do homem com animais de estimação, tais como o alívio de estresse e diminuição da progressão de doenças a ele relacionadas, a redução da ansiedade, a intensificação do desenvolvimento de habilidades socioemocionais e cognitivas (RICCI et al., 2014; DOMINGUES et al., 2015). Entretanto, em contraposição a esta convivência harmoniosa, há a violência contra os animais. Práticas de maus tratos contra estes também têm sido relacionadas à violência interpessoal, fato que acentua a importância do assunto (HOLOYDA; NEWMAN, 2016). Diferentes métodos diagnósticos têm sido empregados na tentativa de identificação de agressões contra os animais (HAMMERSCHMIDT; MOLENTO, 2012). Tendo em vista que, por vezes estas vítimas de maus tratos são encontradas sem vida, ou morrem logo após o resgate, torna-se necessária a realização de exames post-mortem. A necropsia pode ser utilizada nestas situações, e quando associada a exames histopatológicos e toxicológicos são extremamente eficazes tanto na determinação da causa mortis quanto na detecção da existência de maus tratos (GERDIN; MCDONOUGH, 2013).

A conscientização e educação são apontadas como métodos de redução das práticas de maus tratos (DELABARY, 2012). Tendo em vista a ocorrência de maus tratos contra cães e a escassez de trabalhos que visam a determinação de aspectos relacionados às vítimas, este trabalho buscou a identificação das características de cães que tenham sido vítimas de abusos.

\section{MATERIAL E MÉTODOS}

O experimento foi conduzido no período de agosto de 2016 a julho de 2017, e inicialmente desenvolveu-se um questionário com o intuito de obter informações sobre as características de cães que tenham sido vítimas de maus tratos. Os 30 cães deste estudo encontravam-se acolhidos por diferentes indivíduos, entre estes, um tutor, dois protetores independentes, três adotantes, três grupos de protetores, que não faziam parte de nenhuma ONG, e por sete pessoas que se sensibilizaram com a situação do animal que resgataram.

As perguntas presentes no questionário foram de múltipla escolha, entretanto, havia espaço para a descrição de outros tópicos caso houvesse a 
necessidade. O questionário foi dividido em quatro categorias, sendo estas: informações gerais do animal, condições de resgate, condição de saúde do cão e adoção. Na categoria de informações gerais do animal constavam perguntas relacionadas à raça, porte (pequeno, médio, grande), sexo (macho ou fêmea) e idade que continha três opções de resposta, sendo elas: jovem (até um ano de idade), adulto (a partir de um ano até oito anos) e idoso (a partir de oito anos de idade). Em relação às condições de resgate, objetivou-se identificar a origem do animal (rua, tutor, criador e outros) e o tipo de maus tratos.

Em relação à condição de saúde do animal avaliou-se a presença de ectoparasitas (sim ou não), o estado nutricional do animal (bom ou ruim), a presença de lesões macroscópicas, que foram definidas como ferimentos causados por corte, fratura, queimadura ou outros, além disso, verificou-se ainda a existência de dano físico ou funcional permanente (sim ou não) e a descrição do dano.

Por fim averiguava-se a possibilidade de adoção do animal, e no caso de impedimento, qual a razão. Em todos os casos em que se fez necessário a adesão da opção "outros" no questionário, destinou-se um espaço subsequente para a descrição das demais possibilidades.

Além da aplicação do questionário, foram realizados exames anatomopatológicos de cães que com suspeita de maus tratos, visando a determinação da causa da morte do animal e a sua relação com a violência ou negligência para com o mesmo. No caso da constatação de maus tratos, era aplicado 0 questionário de modo a incluí-lo na pesquisa. $O$ estudo anatomopatológico foi conduzido no Setor de Patologia Animal (SPA) da Escola de Veterinária e Zootecnia da Universidade Federal de Goiás (EVZ/UFG).

Fizeram parte do presente estudo 30 casos, caracterizados como maus tratos. A determinação da ocorrência de maus tratos foi baseada nos critérios descritos na legislação, entretanto, os casos deste estudo não obrigatoriamente foram registrados no órgão governamental responsável. Casos de negligência, como a ausência de socorro pós-atropelamento, também foram considerados maus tratos.

Realizou-se a tabulação dos dados obtidos em planilha Excel, e posteriormente estes foram submetidos ao programa "BioEstat 5.3" para a realização das análises estatística. Utilizou-se 0 Teste de Qui-quadrado de aderência, com o intuito de verificar a existência de diferença entre as frequências das seguintes variáveis: raça, porte, sexo, idade, origem do animal, tipo de maus tratos, presença de ectoparasitas, estado nutricional, presença de tumores e presença de lesões macroscópicas. Foi utilizado, neste trabalho, o nível de significância de $5 \%$, ou seja, foram consideradas diferenças significativas quando "p" apresentou valores inferiores a 0,05. Além disso, utilizou-se o Teste-G em duas amostras independentes, para verificar a existência de correlação entre os seguintes dados: porte do animal e faixa etária. Para este teste também foi considerada a existência de independência quando "p" apresentou valores inferiores a 0,05.

\section{RESULTADOS E DISCUSSÃO}

A primeira categoria do questionário, relacionada à resenha do animal permitiu verificar-se que, entre os 30 animais que fizeram parte deste estudo, 22 cães eram Sem Raça Definida (SRD) e somente oito eram de raça, sendo dois ShihTzus, um Poodle, um Samoieda, um Bull Terrier, um Pit bull, um Maltês e um Schnauzer. A quantidade de cães $S R D$ foi significativamente maior em relação à quantidade de cães de raça $(p=0,0176)$. 
A maior quantidade de cães SRD, presentes neste trabalho, pode ser justificada por estudo sobre a dinâmica da população de cães e gatos realizado por Trapp et al. (2015), em uma cidade do sul do Brasil, em que também identificaram maior número de cães SRD comparados a outras raças. Bastos (2013) e Souza e Medeiros (2016), também citam predominância de cães SRD nos municípios brasileiros, fato que de acordo com os autores pode ser justificado pela ausência de um controle reprodutivo significativo, aumentando o risco de abandono.

Em relação ao porte dos animais verificou-se que 10 eram de pequeno porte, 15 de médio porte e quatro de grande porte. A partir dos dados obtidos, notou-se diferença significativa entre o porte dos animais $(p=0,0434)$, com maior número de cães de médio porte quando comparados aos de grande porte $(p=0,0218)$. Entretanto, não foi observada diferença significativa entre os de pequeno porte em relação aos de médio $(p=0,4237)$ e grande porte $(p=0,1814)$, (tabela 1$)$.

TABELA 1 Diferenças entre as frequências dos portes de trinta cães acometidos por maus tratos, entre os anos de 2016 e 2017, Goiânia, Goiás.

\begin{tabular}{c|c}
\hline Porte & $\begin{array}{c}\text { Frequência de } \\
\text { acometimento }\end{array}$ \\
Pequeno & $10^{\mathrm{a}, \mathrm{b}}$ \\
Médio & $15^{\mathrm{a}}$ \\
Grande & $4^{\mathrm{b}}$ \\
\hline *Letras diferentes indicam diferença estatística ao nível \\
de 5\% de significância.
\end{tabular}

Em relação ao porte dos animais que sofreram maus tratos observou-se que os cães mais acometidos foram os de médio e pequeno porte, respectivamente. Estudos que avaliavam o perfil de cães errantes em um local de constante abandono de animais também identificaram haver maior frequência de cães de médio e pequeno porte (KOTVISKI; BURGARDTI, 2014). Como mencionado por Oliveira et al. (2016), as pessoas adquirem um filhote por acharem "engraçadinho", depois que estes crescem, podem ficar maiores do que o esperado ou os tutores perdem o interesse e os abandonam. Normalmente cães de pequeno e médio porte são aparentemente os mais indicados para crianças, apartamentos ou para ficarem dentro de casa, fato que pode explicar o resultado encontrado no presente estudo.

Entre os animais que sofreram maus tratos houve maior frequência de fêmeas $(n=17)$ em relação aos machos $(n=13)$, apesar disso, não foi identificada diferença estatística entre as frequências dos sexos $(p=0,5839)$. Estudos realizados por Rodrigues et al., (2017) identificaram maior população de cadelas domiciliadas no município de Patos de Minas em Minas Gerais. Contrariamente, estudos conduzidos por Soto et al., (2006) identificaram maior percentual de cães machos domiciliados no município de Ibiúna, São Paulo. Logo, é possível observar que a composição sexual da população canina varia de acordo com o município. A ausência de estudos acerca desta composição no município de Goiânia, Goiás, impossibilita uma ponderação mais precisa sobre as frequências sexuais observadas.

Em relação à idade, todos os cães da pesquisa foram categorizados apenas em duas das três opções de faixa etária, jovem $(n=13)$ e adulto $(n=17)$. No entanto, não houve diferença significativa nas categorias de sexo e faixa etária dos animais que fizeram parte deste estudo (ambos, $p=0,5839$ ). Por meio do 
Teste-G de independência, constatou-se que não há relação entre a faixa etária e o porte do animal $(p=1,0000)$. Entretanto, observou-se que dentre os cães vítimas de maus tratos houve maior frequência de adultos de médio porte (8), seguido de adultos de pequeno porte (7) e jovens de médio porte (7).

Quanto à origem, 60\% $(n=18)$ dos cães eram oriundos da rua, $36,66 \%(n=11)$ foram resgatados dos próprios tutores, com $n=11$ e somente um deles, representando 3,33\%, era proveniente de criador de cães de raça, clandestino. Diferença significativa só foi observada em relação ao animal resgatado do criador clandestino $(p=0,0007)$ (tabela 2$)$.

TABELA 2 Diferenças entre as frequências das origens de 30 cães acometidos por maus tratos, entre os anos de 2016 e 2017, Goiânia, Goiás.

\begin{tabular}{l|c}
\hline \multicolumn{1}{c|}{ Origem } & $\begin{array}{c}\text { Frequência de } \\
\text { acometimento }\end{array}$ \\
\hline Rua & $18^{\mathrm{a}}$ \\
Tutor & $11^{\mathrm{a}}$ \\
Criador clandestino & $1^{\mathrm{b}}$ \\
\hline *Letras diferentes indicam estatística ao nível de \\
$5 \%$ de significância.
\end{tabular}

Os dois tipos de maus tratos mais frequente foram o abandono com 18 animais $(60 \%)$, seguido da privação de atendimento médico veterinário em cinco casos $(16,66 \%)$, sendo o primeiro significativamente maior que o segundo tipo. $(p=0,0074)$. Como descrito neste estudo, Alves et al. (2013); Paixão e Machado (2015) mencionam que o abandono de animais é uma prática frequente em todo o mundo, sendo responsável por prejuízos à saúde pública, além de comprometer o bem estar dos animais.

Em relação às condições de saúde dos animais, observou-se que não houve diferença significativa $(p=0,3613)$ entre o número de animais acometidos por ectoparasitos $(n=12 ; 40 \%)$ e aqueles não infestados $(n=18 ; 60 \%)$. Também não foi constatada diferença estatística $(p=0,2012)$ entre o estado nutricional dos cães, sendo que a maioria constituída por $63,33 \%(n=19)$ dos animais exibiam bom estado nutricional, enquanto $36,66 \%(n=11)$ apresentaram estado nutricional ruim.

Apesar de não haver diferença significativa entre o número de animais infestados ou não por ectoparasitos, é importante ressaltar que os ectoparasitos apresentam elevada importância não só pela ação espoliativa exercida, mas também, devido à atuação como vetor de diversas doenças, dentre estas, destacamse as hemoparasitoses, de grande importância clínica e que podem ser transmitidas através da picada de carrapatos (ALMEIDA et al., 2012). Este parece ser um problema oriundo do próprio abandono, uma vez que animais em situação de rua tem maior probabilidade de adquirirem ectoparasitos devido a inexistência de um tratamento profilático adequado.

Em relação à presença de tumores, apenas cinco cães apresentavam nódulos visíveis. Três destes foram diagnosticados como Tumor Venéreo Transmissível (TVT), um tumor de mama, e outro não foi realizada a análise histopatológica. Em relação aos cinco cães que apresentaram tumores, apesar destes poderem ter desenvolvido a enfermidade após estarem na rua, não se pode 
descartar a possibilidade deste ter sido um dos motivos da rejeição já que Baquero et al., (2015) e Magalhães et al., (2016), mencionam que a existência de doenças é uma das principais razões que leva ao abandono do animal pelo tutor.

O TVT, como explicado por Huppes et al. (2014), é transmitido principalmente através do coito, além disso, os animais errantes e sem raça definida são os principais acometidos por essa neoplasia. Deste modo, pressupõe-se que a permissão do acesso à rua ou o período na rua após o abandono configurou um fator determinante para o surgimento da doença.

A maioria dos cães $(n=17)$ não apresentava lesão macroscópica no momento do resgate, no entanto 13 animais possuíam algum tipo de lesão. Não houve diferença significativa $(p=0,5839)$ entre estes dados, e as lesões mais observadas foram as de pele $(n=7)$, corte $(n=2)$, fratura $(n=2)$, oculares $(n=2)$ e queimadura $(n=1)$. Um dos cães, do estudo, apresentou duas lesões simultâneas, sendo uma ocular e outra na pele.

Em relação aos animais lesionados, um dos principais achados imediatos ao resgate foram as lesões dermatológicas, sendo uma afecção bastante comum em cães, podendo compor até $75 \%$ da rotina de atendimento de clínicas, de acordo com Costa et al., (2016). Estudos indicam que condições de estresse crônico podem levar a imunossupressão (CAPRISTE et al., 2017), logo estes animais que por vezes estão com a imunidade reduzida seja por estresse ou até mesmo outros fatores como a dor ou a presença de parasitos, são mais susceptíveis a doenças.

Dos animais avaliados, verificou-se que $17(56,66 \%)$ cães não apresentaram prejuízo físico ou funcional. Entretanto, outros cinco $(16,66 \%)$ permaneceram com algum dano, entre estes problemas permanentes destaca-se claudicação, cegueira, ausência de orelha, cicatriz de queimadura e déficit neurológico. Oito animais vieram à óbito. Destes, quatro morreram em decorrência de hemoparasitose, já outros dois devido à intoxicação, um em função de septicemia decorrente de dermatite atópica, e em um dos casos não houve determinação da causa mortis, pois, o mesmo não foi encaminhado para realização de exame anatomopatológico.

Apesar da maioria dos animais, deste estudo, ter sobrevivido, há a necessidade de se destacar a morte de oito destes, índice superior a $30 \%$, devido à ação do ser humano ao agredir, abandonar e/ou negligenciar cuidados básicos ou veterinários. Este tipo de atitude deve ser combatida através da conscientização da população e quando preciso mediante denúncias e cumprimento da legislação em vigor.

Dentre os 22 animais sobreviventes, 11 já haviam sido adotados, 10 estavam esperando adoção e um deles exibia grande dificuldade para ser adotado devido aos latidos excessivos quando ficava sozinho, comportamento destrutivo e hiperatividade, além disso, de acordo com os responsáveis a aparência do animal também constituía-se em um empecilho para sua adoção.

Nos casos dos cães resgatados por protetores de animais ou ONGs após o resgate e os cuidados/atendimentos especializados necessários o animal fica à disposição para a adoção. No entanto, o adotante deverá praticar a guarda responsável do animal, na qual o tutor assume a reponsabilidade de atender as necessidades psicológicas, físicas e ambientais do cão. Além de se comprometer a atentar-se ao bem-estar do animal e a diminuir riscos que o mesmo poderá levar para a comunidade, como citado por Domingues et al., (2015), o conhecimento das responsabilidades para com 0 animal torna-se um elemento determinante na redução da incidência de maus tratos. Portanto o conhecimento 
prévio das necessidades de cada cão, das implicações de sua aquisição e a verificação da disponibilidade do atendimento às necessidades deste, é um método eficaz na redução do abandono.

\section{CONCLUSÕES}

No presente estudo, no qual foram estudados os casos de 30 cães em situação de maus tratos, foi possível verificar-se que:

- A maioria dos cães era SRD, adulto e de médio e pequeno porte.

- Não houve diferença significativa no número de fêmeas e machos.

- A maioria foi resgatado da rua, provavelmente devido ao abandono, sendo este o tipo de maus tratos mais observado.

- As principais afecções observadas no momento do resgate foram as dermatopatias.

- Mais de $30 \%$ dos cães resgatados vieram a óbito.

\section{REFERÊNCIAS}

ABONIZIO, J.; BAPTISTELLA, E. dos S. T. O papel do consumo na construção de relacionamentos entre humanos e pets, Ponto Urbe [Online], n. 19, p. 1-22, 2016. Disponível em: <http://journals.openedition.org/pontourbe/3257> doi: 10.4000/pontourbe.3257

ALMEIDA, A. do B. P. F. de; PAULA, D. A. J. de; DAHROUG, M. A. A.; FREITAS, A. G. de; SILVA, J. N. da, et al. Ehrlichia canis e Anaplasma platys em carrapatos de cães de Cuiabá, Mato Grosso. Semina: Ciências Agrárias. v. 33, n. 3, p. 11231226, 2012. Disponível em: < http://www.redalyc.org/html/4457/445744113034/> doi: 10.5433/1679-0359.2012v33n3p1123

ALVES A.J.S.; GUILOUX A.G.A.; ZETUN C.B.; POLO G.; BRAGA G.B. et al. Abandono de cães na América Latina: revisão de literatura. Revista de Educação Continuada em Medicina Veterinária e Zootecnia do CRMV-SP, v. 11, n. 2, p. 34 - 41, 2013. Disponível em: <http://www.revistas.bvsvet.org.br/recmvz/article/view/16221>

BAQUERO, O. S.; CHIOZZOTTO, E. N.; GARCIA, R. de C. M.; AMAKU, M.; FERREIRA, F. Demographic characteristics of owned of dogs and cats of Votorantim, São Paulo. Ciência Rural, v. 45, n. 11, p. 2039-2043, 2015. Disponível em: $\quad<h t t p: / / w w w . s c i e l o . b r / s c i e l o . p h p ? s c r i p t=s c i=a r t t e x t \& p i d=S 0103-$ $84782015001102039 \& \operatorname{lng}=$ en\&tIng=en $>$ doi:10.1590/0103-8478cr20141646

BASTOS, A. L. F. Estudo da dinâmica populacional e das estratégias de manejo da população canina no município de Itabirito, MG, Brasil de 2007 a 2011. Tese (Doutorado em Ciência Animal) - Escola de Veterinária, Universidade Federal de Minas Gerais, Belo Horizonte, 2013. Disponível em: <http://www.bibliotecadigital.ufmg.br/dspace/handle/1843/SMOC-

9DWMBP?show=full>

CAPRISTE, M. L. P.; MORAES, N. D. de; SAILER, G. C.; CARDOSO, L.; PRETO, V. A. Reflexões sobre a influência do estresse crônico na transformação de células saudáveis em células cancerígenas. Revista de Enfermagem 
UFPE Online. v.11, n. 6, p. 2473-2479, 2017. Disponível em: <https://periodicos.ufpe.br/revistas/revistaenfermagem/article/view/23412/19084> doi: 10.5205/reuol.10827-96111-1-ED.1106201728

COSTA, G. M.; ARAUJO, S. L.; JÚNIOR, F. A. F. X.; VIANA, D. de A.; EVANGELISTA, J. S. A. M. Dermatological manifestations associated with canine hypothyroidism: A review. Revista Brasileira de Higiene e Sanidade Animal. v. 10, n. $4, \quad$ p. 781-797, 2016. Disponível em: <http://www.higieneanimal.ufc.br/seer/index.php/higieneanimal/article/view/371/1874 > doi: http://dx.doi.org/10.5935/1981-2965.20160064

DELABARY, B. F. Aspetos que influenciam os maus tratos contra animais no meio urbano. Revista Eletrônica em Gestão, Educação e Tecnologia Ambiental, v.5, n.5, p.835-40, 2012. Disponível em: < https://periodicos.ufsm.br/reget/article/viewFile/4245/2813> doi: $10.5902 / 223611704245$

DOMINGUES, L. R.; CESAR, J. A.; FASSA, A. G.; DOMINGUES, M. R. Guarda responsável de animais de estimação na área urbana do município de Pelotas, $R S$, Brasil. Ciência \& saúde coletiva. v. 20, n. 1, p. 185-192, 2015. Disponível em: < https://www.ncbi.nlm.nih.gov/pubmed/25650612> doi: 10.1590/141381232014201.19632013.

GERDIN, J. A.; MCDONOUGH S. P. Forensic pathology of companion animal abuse and neglect. Veterinary Pathology, v.50, n.6, p.994-1006, 2013. Disponível em: < http://journals.sagepub.com/doi/abs/10.1177/0300985813488895> doi: 10.1177/0300985813488895

HAMMERSCHMIDT, J.; MOLENTO, C. F. M. Protocol for expert report on animal welfare in case of companion animal cruelty suspicion. Brazilian Journal of Veterinary Research and Animal Science, v.51, n.4, p.282-96, 2012. Disponível em:

https://www.researchgate.net/publication/276394498_Protocolo_de_pericia_em_bem -estar_animal_para_diagnostico_de_maus-tratos_contra_animais_de_companhia> doi: $1 \overline{0} .11606 /$ issn. $1678-4456 . v 51$ i4p282-296

HOLOYDA, B. J.; NEWMAN, W. J. Childhood animal cruelty, bestiality, and the link to adult interpersonal violence. International Journal of Law and Psychiatry. v. 47, p. 129-135, 2016. Disponível em: https://www.sciencedirect.com/science/article/pii/S0160252716300309?via\%3Dihub> doi: 10.1016/j.ijlp.2016.02.017

HUPPES, R. R.; SILVA, C. G.; USCATEGUI, R. A. R.; DE NARDI, A. B.; F. W. SOUZA, F. W., et al. Tumor venéreo transmissível (tvt): estudo retrospectivo de 144 casos. ARS Veterinaria. v. 30, n. 1, p. 13-18, 2014. Disponível em: < http://arsveterinaria.org.br/index.php/ars/article/view/785> doi: http://dx.doi.org/10.15361/2175-0106.2014v30n1p13-18 
KOTVISKI, B. M.; BURGARDT, S. Densidade e distribuição espacial da população canina encontrada no câmpus de Uvaranas - UEPG, Ponta Grossa, Paraná, Brasil. Estudos de Biologia. v. 36, n. 86, p.1-11, 2014. Disponível em: < https://periodicos.pucpr.br/index.php/estudosdebiologia/article/view/22964/22062> doi: 10.7213/estud.biol.36.086.AO01

MAGALHÃES, C. da S.; LIMA, W. C.; LIMA, D. A. S. D.; QUESSADA, A. M.; DORNELLES, D. E. M., et al. Conhecimento de tutores de cães sobre tumor de mama em cadelas. Acta Veterinaria Brasilica. v. 10, n. 2, p. 186-189, 2016. Disponível em:

https://periodicos.ufersa.edu.br/index.php/acta/article/view/5537/6003>

doi: http://dx.doi.org/10.21708/avb.2016.10.2.5537

OLIVEIRA, A.B.; LOURENÇÃO, C.; BELIZARIO, G.D. Índice estatístico de animais domésticos resgatados da rua vs adoção. Revista Dimensão Acadêmica, v.1, n.2, jul-dez. 2016. Disponível em: <file:///C:/Users/Ana\%20Paula/Desktop/revistadimensao-academica-v01-n02-artigo-01\%20motivos\%20abandono.pdf>

OLLIVER, M. Reconstruire et comprendre l'histoire de la domestication du chien grâce à la paléogénétique. Les nouvelles de l'archéologie, n.148, p. 50-55, 2017. Disponível em: <http://journals.openedition.org/nda/3728\#authors> doi $10.4000 /$ nda. 3728

PAIXÃO, R. L.; MACHADO, J. C. Conexões entre o comportamento do gato doméstico e casos de maus-tratos, abandono e não adoção. Revista Brasileira de Direito Animal. v. 10, n. 20, p. 137-168, 2015. Disponível em: < https://portalseer.ufba.br/index.php/RBDA/article/view/15300> doi: http://dx.doi.org/10.9771/rbda.v10i20.15300

RICCI, G. D.; TORELLI, C.; MARTINS, M. de F.; ALMEIDA, T. W. de Animais solidários: a zooterapia como extensão universitária para idosos institucionalizados. Revista de Cultura e Extensão USP. v. 11, p. 113-121, 2014. Disponível em: < www.producao.usp.br/bitstream/handle/BDPI/45026/MAA_48_2477418_R.pdf?sequ ence=1\&isAllowed $=\mathrm{y}>$ Acesso em: 25 de janeiro de 2018 doi: http://dx.doi.org/10.11606/issn.2316-9060.v11i0p113-121

RODRIGUES, I. M. A.; LUIZ, D. P.; CUNHA, G. N. Princípios da guarda responsável: perfil do conhecimento de tutores de cães e gatos no município de Patos de Minas MG. ARS Veterinaria, v. 33, n. 2, p. 064-070, 2017. Disponível em: < http://www.arsveterinaria.org.br/index.php/ars/article/view/1082/1099> doi: http://dx.doi.org/10.15361/2175-0106.2017v33n2p64-70

SOTO, F. R. M.; FERREIRA, R.; PINHEIRO, S. R.; NOGARI, F.; RISSETO, M. R.; SOUZA, O.; AMAKU, M. Dinâmica populacional canina no Município de Ibiúna - SP: estudo retrospectivo. Brazilian Journal of Veterinary Research in Animal Science. v. 43, n. 2, p. 178-185, 2006. Disponível em: < http://www.revistas.usp.br/bjvras/article/viewFile/26497/28280 > doi: 10.11606/issn.1678-4456.bjvras.2006.26497 
SOUZA, C. C. F.; MEDEIROS, M. A. de Fatores de risco e transtornos comportamentais concomitantes em cães de companhia com medo exagerado a sons. Brazilian Journal of Veterinary Medicine. v. 38, n. 2, p.175-182, 2016. Disponível em: < http://rbmv.org/index.php/BJVM/article/view/193> doi: https://doi.org/10.2430/00000000000000

TRAPP, S. M.; MAEDA, M. S. C. de F.; KEMPER, B.; JUNIOR, F. A. B.; FREIRE, R. L., et al. Population demographic survey and ownership of pet dogs and cats from a small city of southern Brazil. Semina: Ciências Agrárias. v. 36, n. 5, p. 3211-3226, 2015.

Disponível em:

http://www.uel.br/revistas/uel/index.php/semagrarias/article/view/20995/17598> doi: $10.5433 / 1679-0359.2015 v 36 n 5 p 3211$ 